\title{
ANÁLISIS GENÉTICO DE COMPONENTES NUTRICIONALES EN CRUZAS SIMPLES DE MAÍZ DE GRANO BLANCO
}

\section{GENETIC ANALYSIS OF NUTRITIONAL COMPONENTS IN SINGLE CROSSES OF WHITE-KERNEL MAIZE}

\section{Etzael Núñez-Terrones ${ }^{1}$, Ma. del Carmen Mendoza-Castillo*, Adriana Delgado-Alvarado², Fernando Castillo-González ${ }^{1}$ y F. Javier Sánchez-Ramírez ${ }^{1}$}

${ }^{1}$ Colegio de Postgraduados (COLPOS), Campus Montecillo, Montecillo, Texcoco, Estado de México, México. ${ }^{2}$ CoLPOS, Campus Puebla, Santiago Momoxpan, San Pedro Cholula, Puebla, México.

*Autor para correspondencia (camen@colpos.mx)

\section{RESUMEN}

Para proponer el mejor método de mejoramiento genético de una población es indispensable conocer sus parámetros genéticos y la variación genética que en ella existe. El objetivo de este estudio fue identificar los efectos genéticos, así como los posibles efectos maternos que actúan sobre algunos componentes nutrimentales y bioactivos del grano de maíz (Zea mays L.) en cruzas simples formadas a partir de cinco líneas endogámicas $S_{6}-S_{8}$. Las cruzas fueron desarrolladas para sistemas de producción en condiciones de riego y se evaluaron en Montecillo, Texcoco, Estado de México durante el ciclo de producción primavera-verano 2014. Con el grano de la $\mathrm{F}_{1}$ de las cruzas y de los progenitores se realizó el análisis genético del contenido total de aceite, proteína, almidón, cenizas y ácido fítico. Los resultados mostraron mayor variación para la aptitud combinatoria específica (ACE) que para la aptitud combinatoria general (ACG) en cada uno de los componentes nutrimentales; la ACE y ACG representaron, en promedio, 53 y $12 \%$, respectivamente, de la variación entre los genotipos, por lo que los efectos genéticos no aditivos (dominancia y epistasis) prevalecieron sobre los aditivos (ACG) entre los genotipos evaluados. Los efectos maternos y recíprocos mostraron diferencias significativas $(P \leq 0.01)$ para todas las variables, excepto para contenido de almidón. Se definió el comportamiento de los progenitores y de sus cruzas para dirigir el aprovechamiento eficiente de las características evaluadas. Algunas cruzas fueron superiores a sus progenitores en el contenido de los componentes bioquímicos, pero es necesario considerar la dirección del cruzamiento, dados los efectos maternos significativos encontrados en los componentes del grano.

Palabras clave: Zea mays L., componentes nutricionales, grano blanco, ácido fítico.

\section{SUMMARY}

In order to define the best breeding method to improve a population, it is essential to know its genetic parameters and the genetic variation that exists in it. The objective of this study was to identify genetic effects and possible maternal effects acting on some nutritive and bioactive components of maize kernel (Zea mays L.) in single crosses formed from five $\mathrm{S}_{6}-\mathrm{S}_{8}$ inbred lines. The crosses were developed for production systems under irrigation and were evaluated in Montecillo, Texcoco, State of Mexico during the SpringSummer 2014 production cycle. The genetic analysis of total content of oil, protein, starch, ash, and phytic acid was carried out on the $F_{1}$ kernels from the crosses and kernels of the parents. The results showed greater variation for the specific combining ability (SCA) than for general combining ability (GCA) in each of the nutritional components. On average, SCA and GCA represented
53 and $12 \%$, respectively, of the variation between genotypes, hence the non-additive genetic effects (dominance and epistasis) prevailed over the additive ones (GCA) in the genotypes evaluated. Maternal and reciprocal effects showed significant differences $(P \leq 0.01)$ for all traits, except for starch content. The performance of the parents and their crosses was determined to direct the efficient use of the evaluated traits. Some crosses were better than their parents in the content of biochemical components; nevertheless it is necessary to consider the direction of the crossing, given the significant maternal effects found in the components of the kernel.

Index words: Zea mays L., nutritional components, white corn, phytic acid

\section{INTRODUCCIÓN}

El maíz (Zea mys L.) es el componente principal en la dieta de la sociedad mexicana, y su diversidad genética se encuentra ampliamente asociada con la diversidad de formas de consumo y de usos. El grano de maíz es una fuente rica en componentes de la nutrición del humano tales como carbohidratos, proteínas, aceites, minerales y compuestos bioactivos como el ácido fítico (Kirk et al., 1996; Raboy et al., 2001); ésta es la mayor molécula de almacenamiento de fósforo en los cereales, y por sus propiedades para formar quelatos con los cationes minerales como hierro, zinc y calcio, hace difícil su disponibilidad para los animales monogástricos, por lo cual también se le conoce como antinutriente (Raboy et al., 2001), y por lo tanto, es de interés estudiar su presencia en los granos de maíz.

La amplia diversidad genética en este cereal propicia la existencia de una amplia variación en los componentes nutricionales que lo integran, por lo que se considera que su aprovechamiento eficiente, además de mejorar el rendimiento de grano por unidad de superficie, permitiría a la población más vulnerable (en pobreza y pobreza extrema) enfrentar problemas graves de salud y desnutrición (Serna-Saldívar et al., 2013). 
De acuerdo con el tipo de maíz producido en México, el de grano blanco ocupa el primer lugar, al representar $88 \%$ de la producción nacional (SIAP, 2017). Su uso es principalmente para la alimentación humana, aunque de él también pueden obtenerse aceites e insumos para la fabricación de barnices, pinturas, jabones y gomas (Qamar et al., 2016). Referente a su composición, Cázares-Sánchez et al. (2015) señalan que en el grano de maíz la cantidad de proteína y aceite no es alta en comparación con la de otros cereales, pero que existe variación entre y dentro de razas o poblaciones, que debe ser aprovechada en los programas de fitomejoramiento. Figueroa et al. (2013) mencionan que el poco conocimiento del uso y calidad bioquímica del germoplasma ha repercutido en pérdidas de material valioso que podría haber sido integrado en los programas de mejoramiento, por lo que la exploración del material existente en este sentido es muy importante.

En el proceso de mejora de los cultivos, el conocimiento de los componentes genéticos del germoplasma que participa es básico para definir el esquema de mejoramiento adecuado (Esquivel et al., 2013). Entre las herramientas genéticas que proveen información de este tipo se encuentran los diseños de apareamiento, entre los que destacan por su amplio uso los diseños dialélicos (Hallauer et al., 2010); éstos son sistemas de cruzamientos en los cuales un conjunto de $p$ líneas progenitoras y todas las cruzas posibles entre ellas pueden ser evaluadas $\left(p^{2}\right)$. Con base en este principio se desarrollaron cuatro métodos: I) las $p^{2}$ combinaciones posibles, II) los progenitores y las cruzas $F_{1}$ directas, III) las cruzas $F_{1}$ directas y recíprocas y IV) las cruzas $F_{1}$ directas. Frecuentemente, el efecto materno, asociado con ADN citoplásmico del progenitor femenino no se considera al emplear el sistema de cruzas dialélicas (Métodos II y IV); sin embargo, no considerarlo induce un posible error en la estimación del control genético del germoplasma estudiado, cuando se sospecha la presencia de efectos maternos (Ávila et al., 2009; Mahgoub, 2011; Sánchez-Ramírez et al., 2017); por lo que el Método I, donde se consideran todas las combinaciones posibles, incluidas las cruzas recíprocas, será el adecuado.

Los diseños dialélicos permiten conocer el tipo de acción génica que opera en las poblaciones estudiadas a través de la estimación de su capacidad para combinarse (Hallauer et al., 2010); es decir, la aptitud combinatoria general (ACG) y específica (ACE), la primera definida como el comportamiento promedio de una línea en combinaciones híbridas; y la segunda, como aquellos casos donde las combinaciones híbridas se comportan relativamente mejor o peor de lo esperado, con respecto al comportamiento promedio de las líneas involucradas (Sprague y Tatum, 1942). La ACG generalmente se asocia con efectos genéticos aditivos, mientras que la ACE se asocia con efectos genéticos de dominancia y epistasis (Hallauer et al., 2010).

Con base en lo anterior, se planteó la realización del análisis genético de algunos componentes nutricionales con el objetivo de conocer la aptitud combinatoria de un conjunto de progenitores, así como estimar los efectos maternos y recíprocos en sus respectivos cruzamientos a través del Método I de Griffing (1956) para plantear una estrategia de mejoramiento que permita el óptimo aprovechamiento de la riqueza genética de los componentes nutricionales determinados en el grano de maíz blanco.

\section{MATERIALES Y MÉTODOS}

\section{Material genético}

Las cinco líneas y las 20 cruzas simples posibles evaluadas y analizadas forman parte de un programa de mejoramiento genético para condiciones de riego iniciado en la Universidad Autónoma Chapingo por el Dr. Moisés Mendoza Rodríguez. Las cruzas dialélicas del Método I de Griffing fueron hechas en el Campo Agrícola Experimental del Colegio de Postgraduados, Campus Montecillo con la semilla $F_{1}$ obtenida de las cruzas posibles y de las líneas $\left(S_{6}-S_{8}\right)$; en 2014 se estableció una evaluación en campo para conocer el comportamiento agronómico y rendimiento de grano.

\section{Diseño, unidad experimental y manejo del experimento}

Se utilizó un diseño de bloques completos al azar con 4 repeticiones. Las unidades experimentales fueron de un surco de $5 \mathrm{~m}$ de longitud y $0.80 \mathrm{~m}$ de separación entre surcos; en el mismo sitio experimental y bajo el mismo sistema de cultivo se hicieron polinizaciones fraternales a la $F_{1}$, con las cuales se realizaron los análisis bioquímicos correspondientes. Durante el ciclo del cultivo las plantas se mantuvieron sin restricciones hídricas y con la fertilidad recomendada para el sitio experimental. A la cosecha se seleccionaron cinco mazorcas de polinización controlada (cruzas fraternales), sanas y sin daños mecánicos representativas de cada material genético para los análisis respectivos. El análisis de laboratorio se realizó bajo un diseño experimental completamente al azar, por triplicado, excepto para la determinación de aceite (dos repeticiones).

\section{Análisis de la composición del grano}

Las pruebas bioquímicas del grano se realizaron con una mezcla balanceada formada con el grano de la parte media de cinco mazorcas de cada genotipo, según la metodología propuesta por Galicia et al. (2012) para 
pruebas bioquímicas en maíz. El grano se pulverizó en un molino ciclónico (UDY Corporation ${ }^{\circledR}$, Fort Dollins, Colorado, EUA) y la harina se tamizó a través de una malla metálica número 35 para obtener partículas con tamaño de $0.5 \mathrm{~mm}$. El pesaje de las muestras, los reactivos y demás materiales se registró en una balanza analítica $(A \& D \AA$, Modelo GR 202, San Jose, California, EUA).

Los análisis y métodos empleados fueron: para aceite 30-25.01 (AACC, 1999a); proteína Kjeldahl: 46-11.02 (AACC, 1999b); almidón (Brunt et al., 1998) y 76-13.01 (AACC, 1999c); cenizas 08-01.01 (AACC, 1999d) y humedad con termobalanza (Ohaus ${ }^{\circledR}$ MB 45, Parsippany, Nueva Jersey, EUA).

\section{Determinación de ácido fítico}

El ácido fítico se determinó con el método descrito por Dragičević et al. (2011). En frascos de $50 \mathrm{~mL}$ con tapa se colocaron $250 \mathrm{mg}$ de muestra, se adicionaron $10 \mathrm{~mL}$ de ácido tricloroacético 5 \% y se sometieron a agitación por $1 \mathrm{~h}$. Posteriormente, se extrajeron $5 \mathrm{~mL}$ de solución y se colocaron en tubos Falcon de $15 \mathrm{~mL}$ para centrifugar (centrífuga Hermle ${ }^{\circledR}$ Z $326 \mathrm{~K}$, Wehingen, Alemania) a $12,000 \times g$ durante 15 min a $4{ }^{\circ} \mathrm{C}$. Se realizó una dilución 1:6 con agua desionizada. Para la determinación colorimétrica se tomaron $1.5 \mathrm{~mL}$ de la dilución y se añadieron $0.5 \mathrm{~mL}$ de reactivo de Wade $\left(0.3 \mathrm{~g}\right.$ de $\mathrm{FeCl}_{3} 6 \mathrm{H}_{2} \mathrm{O}+3 \mathrm{~g}$ de ácido 5-sulfosalicílico), aforado a $1 \mathrm{~L}$ con agua desionizada, enseguida se centrifugó a $12,000 \times \mathrm{g}$ por $10 \mathrm{~min}$ a $4{ }^{\circ} \mathrm{C}$. La lectura de absorbancia se realizó a $500 \mathrm{~nm}$ en un espectrofotómetro UV/VIS (Thermo Scientific ${ }^{\circledR}$ Modelo Evolution 300, Waltham, Massachusetts, EUA). Los resultados se expresaron en $\mathrm{g} 100 \mathrm{~g}^{-1}$ de materia seca (ms), a partir de una curva estándar $\left(y=0.0039 x+0.7277, R^{2}=\right.$ 0.970 ) preparada con ácido fítico (Sigma Aldrich ${ }^{\circledR}$, USA).

\section{Análisis estadístico}

Para estimar la aptitud combinatoria general (ACG) y específica (ACE), los efectos maternos (EfMat) y los efectos recíprocos (EfRec) de los genotipos, se realizó el análisis genético basado en el diseño dialélico Método I (Griffing, 1956) con el modelo de efectos fijos, así como pruebas de F propuestas por Martínez (1983) para el diseño correspondiente. El análisis estadístico se realizó con el programa SAS® versión 9.1 (SAS Institute, 2003).

\section{RESULTADOS Y DISCUSIÓN}

\section{Análisis genético}

En el Cuadro 1 se muestran los cuadrados medios del análisis de varianza. Se encontraron diferencias significativas entre genotipos para todas las variables estudiadas. Las diferencias estuvieron asociadas a la variación entre los genotipos, al tipo de acción génica presente, así como a la expresión de heterosis entre las cruzas (Esquivel et al., 2013; Guerrero-Guerrero et al., 2012; Sánchez-Ramírez et al., 2017).

Al descomponer la fuente de variación de genotipos en su ACG y ACE, se encontraron diferencias significativas para los componentes estudiados. De acuerdo con los valores de la suma de cuadrados del análisis de varianza (datos no mostrados), la contribución a la varianza de las características bioquímicas del grano evaluadas, atribuible a los genotipos estuvo explicada por la ACE en $53 \%$, ACG en $12 \%$, EfRec en $20 \%$ y EfMat en $15 \%$; esto indicó que los efectos genéticos de dominancia y epistasis (ACE) fueron de mayor importancia en la concentración de los compuestos bioquímicos estudiados, en comparación con los efectos aditivos (ACG).

En componentes bioquímicos determinados en el grano de maíz y analizados bajo el Método II dialélico, Bisen et al. (2017) encontraron también que los efectos no aditivos fueron de mayor importancia que los aditivos. Estos resultados contrastan con los reportados por PicónRico et al. (2018) en maíz, en donde los efectos aditivos fueron mayores para contenido de aceite en el grano. El contraste de resultados puede asociarse a dos posibles causas, la primera como resultado de las fases de selección previa al desarrollo de las líneas, que en el caso del presente estudio, primero se buscó agotar la varianza aditiva en las poblaciones de origen para posteriormente aprovechar la varianza no aditiva a través de las líneas, y la segunda, mencionada por Mahgoub (2011), donde la no consideración de las cruzas recíprocas puede conducir a una estimación sesgada de los efectos genéticos, particularmente en características asociadas con los componentes del grano.

Los efectos maternos (EfMat) y recíprocos (EfRec) fueron altamente significativos para todas las características, excepto para almidón; es decir, los valores obtenidos en las cruzas directas $(i, j)$ fueron diferentes a los obtenidos en las cruzas recíprocas $(j, i)$. Lo anterior indica que, para la producción de semilla de alguna cruza en particular, referente al incremento o reducción en el contenido de alguno de estos compuestos, se debe considerar específicamente las líneas que fungirán como progenitor femenino o masculino.

\section{Aptitud combinatoria general}

Los valores de los efectos de ACG $\left(g_{j}\right)$ de las líneas se muestran en el Cuadro 2. En contenido de aceite, las líneas 
Cuadro 1. Cuadrados medios del análisis de varianza para características nutricionales en un sistema de cruzas dialélicas completo, desarrollado entre cinco líneas de maíz, evaluadas en condiciones de riego en los Valles Altos Centrales de México.

\begin{tabular}{|c|c|c|c|c|c|c|}
\hline Fuente & $\mathrm{GL}$ & Aceite & Proteína & Almidón & Cenizas & Ácido fítico \\
\hline Repeticiones & $1 / 2^{+}$ & 0.002 & 1.280 & 41.42 & 0.028 & 0.021 \\
\hline Genotipos & 24 & $1.299 * \star$ & $5.100 * \star$ & $69.52 *$ & $0.395 * *$ & $0.367 * \star$ \\
\hline ACG & 4 & $0.400 * \star$ & $5.090 * \star$ & $64.83 \mathrm{~ns}$ & $0.323 *$ & $0.247 \star \star$ \\
\hline ACE & 10 & $1.798 * \star$ & $5.970 \star \star$ & $82.66 *$ & $0.583 * \star$ & $0.410 * \star$ \\
\hline EfMat & 4 & $2.505 * \star$ & $8.620 * \star$ & $47.99 \mathrm{~ns}$ & $0.114 \star \star$ & $0.337 \star \star$ \\
\hline EfRec & 6 & $0.366 * *$ & $1.300 * *$ & $65.12 \mathrm{~ns}$ & $0.319 * *$ & $0.395 * \star$ \\
\hline Error & $24 / 48^{+}$ & 0.094 & 0.370 & 33.31 & 0.010 & 0.018 \\
\hline CV (\%) & & 5.770 & 8.320 & 6.95 & 6.790 & 6.410 \\
\hline
\end{tabular}

GL: grados de libertad, ${ }^{+}$: izquierda, grados de libertad para la variable aceite, derecha, grados de libertad para el resto de variables, ACG: aptitud combinatoria general, ACE: aptitud combinatoria especifica, EfMat: efectos maternos, EfRec: Efectos recíprocos, CV: Coeficiente de Variación; *: significativo $(P \leq 0.05), * *$ : altamente significativo $(P \leq 0.01)$, ns: no significativo estadísticamente.

L2, L3 y L4 tuvieron valores positivos de ACG, aunque sólo en L2 fueron significativos; también fueron estas líneas las que mostraron los mayores valores per se para este

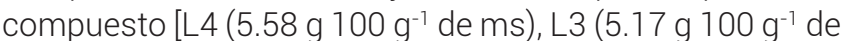
ms) y L2 (5.10 g $\left.\left.100 \mathrm{~g}^{-1} \mathrm{de} \mathrm{ms}\right)\right]$.

Los valores de ACG fueron relativamente bajos para todas las variables de estudio. Al respecto, SánchezRamírez et al. (2017) asociaron este comportamiento con el método utilizado en la selección de las líneas altamente endogámicas (mediante prueba per se y no con pruebas previas de ACG). Bajo este supuesto, las líneas se clasificaron con efectos positivos o de ACG alta $(\mathrm{L} 3, \mathrm{~L} 4, \mathrm{~L} 2)$ o con efectos negativos o de ACG baja ( $L 5$ y L 1 ).
Para el contenido de proteína y de cenizas, las líneas L3 y L4 mostraron efectos positivos y significativos de ACG, con valores de 0.34 y 0.33 para proteína y de 0.07 y 0.08 para cenizas, respectivamente. En contenido de proteína, ninguna de las dos líneas figuró como la de contenido mayor per se entre todos los progenitores, con valores de

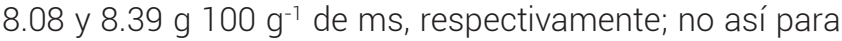
cenizas, donde L4 y L3 tuvieron el mayor contenido entre

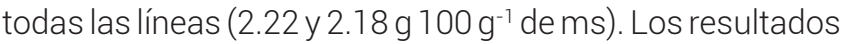
indican que las líneas poseen una porción considerable de genes de efectos aditivos que se expresan favorablemente en el grano, lo que permitiría incrementar su contenido a través de esquemas de selección recurrente.

Cuadro 2. Contenido nutricional (per se) y efectos de aptitud combinatoria general $\left(g_{i}\right)$ de cinco líneas de maíz evaluadas en condiciones de riego en Valles Altos de México.

\begin{tabular}{lcccccccccc}
\hline Línea & Aceite $^{+}$ & $g_{i}$ & Proteína $^{+}$ & $g_{i}$ & Almidón $^{+}$ & $g_{i}$ & Cenizas $^{+}$ & $g_{i}$ & $\begin{array}{c}\text { Ácido } \\
\text { fítico }^{+}\end{array}$ & $g_{i}$ \\
\hline L1 & 3.44 & -0.07 & 7.43 & $-0.67 *$ & 83.40 & 1.35 & 1.14 & $-0.05 *$ & 1.76 & 0.04 \\
L2 & $5.10 *$ & $0.17 *$ & 8.29 & 0.01 & 76.39 & -1.43 & 1.45 & $0.06 *$ & $2.09 *$ & 0.01 \\
L3 & $5.17 *$ & 0.01 & 8.08 & $0.34 *$ & 79.21 & -1.27 & $2.18 *$ & $0.07 *$ & $2.22 *$ & $-0.08 *$ \\
L4 & $5.58 *$ & 0.08 & 8.39 & $0.33 *$ & 75.92 & -0.38 & $2.22 *$ & $0.08 *$ & $2.83 *$ & $0.13 *$ \\
L5 & 3.55 & $-0.19 *$ & $9.62 *$ & -0.01 & 76.48 & 1.74 & 1.51 & $-0.16 *$ & $2.26 *$ & $-0.09 *$ \\
DSH $_{0.05}$ & 0.68 & 0.256 & 1.02 & 0.395 & 9.88 & 3.751 & 0.13 & 0.068 & 0.17 & 0.087 \\
\hline
\end{tabular}

${ }^{{ }^{\prime} V a l o r e s ~ p e r ~ s e ~ e x p r e s a d o s ~ e n ~} \mathrm{~g} 100 \mathrm{~g}^{-1} \mathrm{de} \mathrm{ms}$, *: significativo $(\mathrm{P} \leq 0.05)$, DSH: diferencia significativa honesta. 
La línea L5 mostró efectos negativos para cuatro de las cinco variables evaluadas, lo que podría repercutir en que los cruzamientos que involucren a esta línea, podrían expresar poco potencial de incremento en el contenido de compuestos bioquímicos; no obstante, si lo que se desea es contar con líneas de maíz que reduzcan la concentración de ácido fítico en el grano para consumo humano, debido a la incapacidad de los organismos monogástricos para asimilar este compuesto (Raboy et al., 2001), esta línea, junto con L3, podrían representar fuentes valiosas por su valor negativo y significativo de ACG.

En general, las líneas L3 y L2 mostraron buena respuesta para cuatro de los cinco componentes nutrimentales, por lo que se esperaría que las cruzas que involucren a estos progenitores presentaran valores altos de ACE y de los compuestos bioquímicos estudiados. Se encontró que el contenido de cenizas y de aceite estuvieron directamente asociados con el efecto $g_{i}$; por ejemplo, la línea $L 4$ tuvo

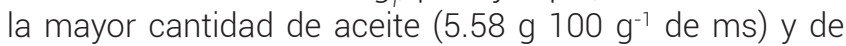
cenizas (2.22 g $100 \mathrm{~g}^{-1}$ de $\left.\mathrm{ms}\right)$, con valor alto de ACG (0.08, por igual), mientras que la línea L 1 tuvo el menor contenido de aceite (3.44 g $100 \mathrm{~g}^{-1}$ de $\mathrm{ms}$ ) y bajo efecto de ACG (-0.07).

\section{Aptitud combinatoria específica}

Los efectos de ACE para los compuestos bioquímicos de las cruzas posibles se muestran en el Cuadro 3. Las cruzas que expresaron los contenidos superiores de aceite, proteína, cenizas, almidón y ácido fítico fueron diferentes entre sí. En aceite no se encontraron diferencias estadísticas significativas entre las 10 cruzas de mayor contenido, cuyo intervalo de variación fluctuó entre 6.83 y

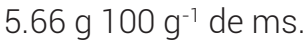

En las cruzas de contenido mayor de aceite estuvieron involucrados los progenitores $L 2$, $L 3$ y $L 4$, que fungieron como hembras o como machos, con altos efectos de ACG y $s_{i j}$ positivos (excepto $L 4 \times L 3$, con un valor de -0.01 ). La cruza $L 5 \times L 1$ formada por los progenitores L5 y L1, de baja ACG, resultó ser la de mayor contenido de aceite (6.83 g $100 \mathrm{~g}^{-1}$ de $\mathrm{ms}$ ), debido quizá a su valor alto y positivo de $s_{i j}$ como señalan Escorcia-Gutiérrez et al. (2010). Las cruzas con bajo contenido de aceite no necesariamente involucraron progenitores con baja ACG o presentaron efectos negativos de ACE $(L 5 \times L 4$ y L3 $\times L 1)$.

Para proteína no se encontraron diferencias estadísticas significativas entre las 12 cruzas de mayor contenido, cuyo intervalo de variación osciló entre 7.21 y 8.95 g 100 $\mathrm{g}^{-1}$ de $\mathrm{ms}$. Destacaron tres progenitores que intervinieron en las cruzas de mayor contenido de proteína (L2, L3 y L4) que participaron como machos o hembras. Los

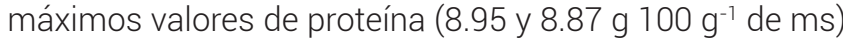

no necesariamente se relacionaron con $\mathrm{s}_{i j}$ altos $(-0.11 \mathrm{y}$ -0.43), aun cuando se originaron de progenitores con alta ACG ( $L 3 \times L 4$ y $L 2 \times L 4)$. Las cruzas de más bajo contenido $(L 4 \times L 2, L 4 \times L 1$ y $L 5 \times L 1)$ presentaron valores bajos de $\mathrm{S}_{i j}(-0.43,-0.38$ y -1.67 , respectivamente). Picón-Rico et al. (2018) reportaron resultados similares y no encontraron consistencia entre valores altos de ACG de los progenitores con valores altos de ACE de sus cruzas.

Algunos estudios señalan que una cruza simple tendrá altos rendimientos de grano si sus dos líneas progenitoras, o al menos una de ellas son de alta ACG y el efecto ACE de la cruza es alto (Escorcia-Gutiérrez et al., 2010); también, en los casos donde las cruzas de rendimiento alto estén formadas con líneas de ACG baja, pero con efectos de ACE positivos y altos (Ávila et al., 2009; GuerreroGuerrero et al., 2012; Sánchez-Ramírez et al., 2017). En este estudio las líneas con alta ACG no necesariamente produjeron las mejores combinaciones híbridas para los caracteres evaluados, debido a que generalmente, como lo mencionaron Picón-Rico et al. (2018), el comportamiento específico de las cruzas no puede ser predicho totalmente con base en los estimadores de ACG de sus parentales; además, el efecto genético extranuclear puede estar incidiendo en las características.

Resultados similares fueron reportados por CervantesOrtiz et al. (2007) en trabajos realizados sobre algunas características de plántula y de planta adulta de maíz, donde líneas con alta ACG no precisamente produjeron buenas combinaciones híbridas para los caracteres evaluados. Por su parte, Long et al. (2004), en estudios sobre biofortificación mineral (hierro y zinc) en grano de maíz, no encontraron relaciones favorables de los efectos positivos o negativos de ACE, independientemente de si los híbridos se formaron a partir de la combinación de progenitores con ACG alta $\times$ alta, alta $\times$ baja o baja $\times$ baja.

Aunque en contenido de proteína (Cuadro 3) se presentaron valores superiores al promedio general (Cuadro 5), ello no implica que estas cruzas sean maíces de alta calidad de proteína (QPM), puesto que se desconoce la proporción y tipo de aminoácidos presentes. En poblaciones QPMs de México se han reportado contenidos

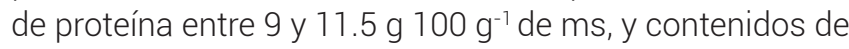
lisina y triptofano del orden de 3.5 a 4.8 y 0.87 a $1.18 \mathrm{~g} 100$ $\mathrm{g}^{-1}$ de proteína, respectivamente (Vázquez et al., 2012).

Debido a que ésta fue una exploración preliminar de las características bioquímicas de grano, no se abordó el estudio puntual de los aminoácidos; aunque existe el interés de conocer la composición detallada de la proteína de estos genotipos para diseñar de mejor manera un programa de mejoramiento genético, por lo que deberá 
diseñarse un estudio específico para tal fin.

Con respecto al contenido de almidón; en general, las cruzas con mayor contenido presentaron valores altos de $s_{i i}$. Entre las cuatro cruzas con mayor contenido de almidón estuvo la línea L5, como progenitor macho o hembra. Las dos cruzas superiores en almidón fueron L5

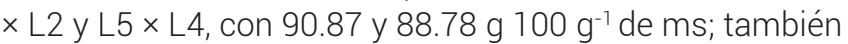
resultaron ser las de mayor efecto de ACE, con 4.56 y 4.40 , respectivamente. El valor más bajo de $s_{i j}$ lo presentaron las cruzas $L 5 \times L 1$ y $L 2 \times L 3$ y sus cruzas recíprocas, con -0.20 $y-2.71$.

Para contenido de cenizas no hubo diferencias estadísticas significativas entre las nueve cruzas de más alta concentración, cuyo intervalo de variación fluctuó

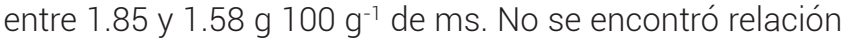
entre los valores altos de ACE con las cruzas superiores; sin embargo, las cruzas de contenido menor sí mostraron valores bajos de $s_{i j}$. La cruza L5 $\times$ L1, con el contenido mayor de minerales en el grano $\left(1.85 \mathrm{~g}^{100 \mathrm{~g}^{-1}} \mathrm{de} \mathrm{ms}\right)$ estuvo formada por dos líneas de baja ACG para esta variable, pero fue la cruza de más alto efecto de $s_{i j}$ con un valor de 0.32. El efecto y contenido de cenizas más bajo lo presentó la cruza $\mathrm{L} 5 \times \mathrm{L} 3$, con -0.54 y $0.70 \mathrm{~g} 100 \mathrm{~g}^{-1} \mathrm{de}$ ms. En comparación con valores de cenizas reportados en la literatura (0.81 g $100 \mathrm{~g}^{-1}$ de ms, Qamar et al., 2016; 1.80 g $100 \mathrm{~g}^{-1}$ de ms, Cázares-Sánchez et al., 2015), algunos genotipos analizados en el presente estudio pueden considerarse como de alto contenido mineral.

Para ácido fítico se encontraron diferencias estadísticas significativas a partir del segundo genotipo de mayor contenido de este compuesto bioquímico. La línea L1 participó en las cinco cruzas de mayor contenido como hembra o como macho, con valores positivos de $s_{i j}$ por lo que es probable que las cruzas que involucren a este progenitor puedan mostrar incrementos sustantivos en el contenido de ácido fítico en el grano. Lo anterior puede ser importante en el área de ganadería, ya que, si los piensos se suplementan con fitasa, con el uso de estas cruzas de alta producción de ácido fítico se podría aumentar la

Cuadro 3. Efectos de aptitud combinatoria específica $\left(s_{i j}\right)$ de las cruzas de mayor a menor contenido de aceite, proteína, almidón, cenizas y ácido fítico en el grano de maíz ( $\left.100 \mathrm{~g}^{-1} \mathrm{de} \mathrm{ms}\right)$.

\begin{tabular}{|c|c|c|c|c|c|c|c|c|c|c|c|c|c|c|}
\hline uza & Aceite & $S_{i j}$ & Cruza & Proteína & $s_{i j}$ & Cruza & Almidón & $s_{i j}$ & Cruza & Cenizas & $S_{i j}$ & Cruza & $\begin{array}{l}\text { Ácido } \\
\text { fítico }\end{array}$ & $s_{i j}$ \\
\hline$\times \mathrm{L1}$ & $83 *$ & $84 *$ & $L 3 \times L 4$ & $895 *$ & -0.11 & $\mathrm{~L} 5 \times \mathrm{L} 2$ & 90.87 & $4.56 *$ & $\mathrm{~L} 5 \times \mathrm{L} 1$ & $1.85 *$ & $0.32 *$ & $\mathrm{~L} 1 \times \mathrm{L} 2$ & $2.75 *$ & $0.19 *$ \\
\hline$x \mathrm{~L} 3$ & $6.48 *$ & $0.32 \star$ & $L 2 \times L 4$ & $8.87 *$ & $-0.43 *$ & $\mathrm{~L} 5 \times \mathrm{L} 4$ & 78 & 40 & $\mathrm{~L} 1 \times \mathrm{L} 3$ & 33* & .01 & L1 & $2.36 *$ & $.10 *$ \\
\hline$\times \mathrm{L} 5$ & $6.19 *$ & $0.30 *$ & $L 3 \times L 5$ & $8.53 *$ & $0.18 *$ & $\mathrm{~L} 1 \times \mathrm{L} 5$ & 75 & -0.20 & $\mathrm{~L} 2 \times \mathrm{L} 4$ & $2 *$ & 0.12 & & 2.33 & $.02 *$ \\
\hline$\times \quad<5$ & $5.99 *$ & $0.58 *$ & $L 4 \times L 5$ & $9 *$ & $0.54 *$ & $L 4 \times L 5$ & 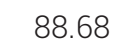 & 40 & L2 & 9* & 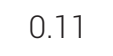 & & 30 & $0.10 *$ \\
\hline$\times \mathrm{L} 1$ & $5.93 *$ & 0.18 & $L 5 \times\llcorner 4$ & $5 *$ & $0.54 \star$ & $L 4 \times L 2$ & & 1.72 & $\mathrm{~L} 2 \times \mathrm{L} 5$ & 1 * & $.16 *$ & & 22 & $0.07 *$ \\
\hline$\times \mathrm{L} 3$ & $5.93 *$ & -0.01 & $\mathrm{~L} 3 \times \mathrm{L} 2$ & $7.85 *$ & 0.05 & $\mathrm{~L} 3 \times \mathrm{L} 1$ & 8 & 2.43 & $\mathrm{~L} 2 \times \mathrm{L} 3$ & 0* & 0.02 & L5 & 2.22 & $0.20 *$ \\
\hline$\times \mathrm{L} 2$ & $5.92 *$ & $0.39 *$ & $L 1 \times L 4$ & 7.81 * & $-0.38 *$ & $\mathrm{~L} 5 \times \mathrm{L} 3$ & 87.37 & 1.22 & $\llcorner 4 \times\llcorner 3$ & $1.62 *$ & -0.02 & L2 & 2.15 & $0.20 *$ \\
\hline $3 \times L 2$ & $5.86 *$ & 0.19 & $\mathrm{~L} 2 \times \mathrm{L} 3$ & $7.67 *$ & 0.05 & $\mathrm{~L} 2 \times \mathrm{L} 1$ & & 0.16 & L3 & $1.62 *$ & -0.02 & & 15 & -0.01 \\
\hline$\times \mathrm{L} 2$ & $5.74 *$ & $0.58 *$ & & & 0 & & & $4.56 *$ & & 8* & & & 12 & -0.26 \\
\hline $2 \times L 1$ & $5.66 *$ & $0.39 \star$ & $L 1 \times L 2$ & 7.32* & $0.80 *$ & $L 4 \times L 1$ & 79 & -0.11 & $\mathrm{~L} 2 \times \mathrm{L} 1$ & 1.45 & 0.11 & $\mathrm{~L} 3 \times \mathrm{L} 5$ & 2.08 & -0.25 \\
\hline $2 \times \mathrm{L} 3$ & 5.49 & 0.19 & $\mathrm{~L} 3 \times \mathrm{L} 1$ & $7.30 *$ & $-0.16 *$ & $L 4 \times L 3$ & 84.44 & 0.28 & $\mathrm{~L} 1 \times \mathrm{L} 4$ & 1.45 & -0.15 & $\mathrm{~L} 3 \times \mathrm{L} 4$ & 2.06 & -0.01 \\
\hline LL & 5.36 & $-0.57 *$ & & & & & & 2.43 & & & & & 05 & -0.35 \\
\hline$\times\llcorner 4$ & 5.06 & 0.18 & L4 $\times$ L3 & 6.89 & -0.11 & $\mathrm{~L} 5 \times \mathrm{L} 1$ & & -0.20 & & & $0.32 *$ & & .95 & $0.07 *$ \\
\hline$\times \mathrm{L} 5$ & 4.95 & $0.84 *$ & $L 1 \times L 3$ & 6.42 & $-0.16 *$ & $\mathrm{~L} 1 \times \mathrm{L} 4$ & 82.89 & -0.11 & $\llcorner 4 \times\llcorner 5$ & 1.36 & $-0.28 *$ & $\mathrm{~L} 3 \times \mathrm{L} 2$ & 1.93 & -0.13 \\
\hline $3 \times L 5$ & 4.88 & $-0.34 *$ & $\mathrm{~L} 1 \times \mathrm{L} 5$ & 6.17 & $-1.67 *$ & $\mathrm{~L} 3 \times \mathrm{L} 5$ & & 1.22 & $\llcorner 4 \times L 1$ & 31 & 15 & & .86 & $0.19 *$ \\
\hline$B \times\llcorner 4$ & 4.86 & -0.01 & $\mathrm{~L} 2 \times \mathrm{L} 5$ & & $-1.33 *$ & $\mathrm{~L} 2 \times \mathrm{L} 3$ & & -2.71 & $\llcorner 4 \times\llcorner 2$ & 1.20 & -0.12 & $\mathrm{~L} 2 \times \mathrm{L} 3$ & .79 & $-0.13 x$ \\
\hline $5 \times L 4$ & 4.79 & $0.30 *$ & $L 5 \times L 2$ & 5.98 & $-1.33 *$ & $\mathrm{~L} 1 \times \mathrm{L} 2$ & 78.86 & 0.16 & $\mathrm{~L} 3 \times \mathrm{L} 1$ & & -0.01 & $\mathrm{~L} 2 \times \mathrm{L} 4$ & .77 & -0.26 \\
\hline$L 5 \times L 3$ & 4.70 & $-0.34 *$ & $L 4 \times L 2$ & 5.66 & $-0.43 *$ & $L 3 \times L 4$ & 78.78 & 0.28 & $\llcorner 3 \times\llcorner 5$ & 1.02 & $-0.54 \star$ & $\mathrm{L} 1 \times \mathrm{L} 5$ & 1.74 & $0.02 *$ \\
\hline $3 \times$ L1 & 4.65 & $0.32 *$ & $L 4 \times L 1$ & 5.45 & $-0.38 *$ & $\mathrm{~L} 2 \times \mathrm{L} 4$ & 78.02 & 1.72 & $\llcorner 5 \times\llcorner 4$ & 0.90 & $-0.28 *$ & $\llcorner 5 \times\llcorner 4$ & 1.45 & -0.35 \\
\hline $2 \times\llcorner 4$ & 4.61 & $-0.57 \star$ & $L 5 \times L 1$ & 3.85 & $-1.67 \star$ & $\mathrm{L} 3 \times \mathrm{L} 2$ & 73.37 & -2.71 & $L 5 \times L 3$ & 0.70 & $-0.54 *$ & $\mathrm{~L} 5 \times \mathrm{L} 3$ & 1.21 & -0.25 \\
\hline $\mathrm{SH}_{0.05}$ & 1.74 & 0.622 & & 1.80 & 0.952 & & 17.45 & 9.04 & & 0.33 & 0.164 & & 0.41 & 0.21 \\
\hline
\end{tabular}

DSH: diferencia significativa honesta; *: significativo $(\mathrm{P} \leq 0.05)$. 
biodisponibilidad de fósforo en los animales, además de que se reduciría la contaminación por fósforo en las áreas con unidades ganaderas bajo manejo intensivo (Vats y Banerjee, 2004). Contrariamente, las cruzas L5 x L4 y L5 $\times$ L3 tuvieron los contenidos menores de ácido fítico con 1.45

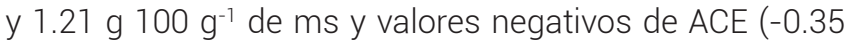
y -0.25 , respectivamente), lo que resulta favorable para el consumo humano y de otras especies monogástricas que están imposibilitadas para metabolizar eficientemente este compuesto (Raboy et al., 2001).

Con los resultados del presente estudio se logró establecer que la expresión del mayor contenido de cada variable no estuvo necesariamente asociada con valores altos de $s_{i j}$ sobre todo en las variables contenido de proteína y de minerales.

\section{Efectos maternos}

Los efectos maternos de los progenitores se presentan en el Cuadro 4, donde se observa que los valores positivos en contenido de aceite de las líneas L4 y L5 indican que tales progenitores expresan su mayor potencial para producir aceite en sus cruzas directas; es decir, cuando estas líneas se usan exclusivamente como progenitor femenino. Las líneas L1, L2 y L3 tuvieron valores negativos, por lo que se espera que se comporten de forma contraria a esta situación. La línea L2 resultó un buen progenitor femenino, pues demostró efectos positivos para proteína (0.35), almidón (0.11), cenizas (0.07) y con efecto materno negativo para la producción de ácido fítico $(-0.13)$, lo que indica que empleada como hembra, su progenie mostrará detrimento en esta característica, pero con altos valores en las otras variables.

\section{Estructura genética}

Para este análisis se consideraron los cinco cruzamientos de mayor y los dos de menor contenido de cada uno de los compuestos bioquímicos evaluados (Cuadro 5). Con base en los valores de los efectos genéticos, la estructura genética de los compuestos en los cruzamientos no presentó tendencias claras.

De acuerdo con los valores genotípicos para el contenido de aceite, las cruzas sobresalientes podrían ser empleadas como híbridos, ya que los efectos de $g_{i}+g_{j}<s_{i j}$; es decir, que los efectos aditivos fueron menores que los no aditivos; entonces, se esperaría que estas cruzas mostraran cambios aleatorios en generaciones posteriores $\left(F_{n}\right)$. Para proteína, las tres cruzas más rendidoras mostraron efectos $g_{i}+g_{j}>s_{i j}$ por lo que estas combinaciones genéticas posiblemente no presenten depresión endogámica significativa en sus generaciones avanzadas, pudiendo utilizarse como variedades de polinización libre o formar con ellas una variedad sintética. Las cruzas $L 4 \times L 5$ y $L 5 \times$ $L 4$ mostraron valores $g_{i}+g_{j}<S_{i j}$, por lo que también podrían ser aprovechadas como híbridos.

En general, para contenido de almidón, los efectos no aditivos fueron de mayor importancia que los aditivos; por lo tanto, las líneas en estudio podrían usarse para la formación de híbridos productores de almidones. Para contenido de cenizas se presentó variación en cuanto al efecto predominante en las cruzas $L 5 \times L 1, L 1 \times L 2$ y $L 2 \times L 5$, pues mostraron efectos $g_{i}+g_{i}<s_{i i^{\prime}}$ mientras que L1 $\times$ L3 y L2 $\times$ L4 tuvieron efectos $g_{i}+g_{j}>s_{i j}$, por lo que, en un programa de mejoramiento para incrementar esta variable, algunos genotipos tendrían que ser manejados

Cuadro 4. Contenido nutricional (per se) y efectos maternos $\left(m_{\mathrm{i}}\right)$ de cinco líneas de maíz evaluadas en condiciones de riego en Valles Altos del Centro de México.

\begin{tabular}{lcccccccccc}
\hline Línea & Aceite $^{+}$ & $m_{i}$ & Proteína $^{+}$ & $m_{i}$ & Almidón & $m_{i}$ & Cenizas $^{\dagger}$ & $m_{i}$ & $\begin{array}{c}\text { Ác. } \\
\text { Fítico }^{+}\end{array}$ & $m_{i}$ \\
\hline L1 & 3.44 & -0.07 & 7.43 & 0.35 & 83.40 & -0.88 & 1.14 & 0.06 & 1.76 & 0.05 \\
L2 & 5.10 & -0.12 & 8.29 & 0.35 & 76.39 & 0.11 & 1.45 & 0.07 & 2.09 & $-0.13^{*}$ \\
L3 & 5.17 & $-0.24 \star$ & 8.08 & 0.44 & 79.21 & -1.53 & 2.18 & -0.04 & 2.22 & 0.07 \\
L4 & 5.58 & $0.41 *$ & 8.39 & $-0.75 *$ & 75.92 & 1.72 & 2.22 & -0.03 & 2.83 & $0.11 *$ \\
L5 & 3.55 & 0.01 & 9.62 & -0.39 & 76.48 & 0.58 & 1.51 & -0.06 & 2.26 & -0.09 \\
DSH $_{0.05}$ & & 0.61 & & 0.988 & & & & 0.17 & & 0.218 \\
\hline
\end{tabular}

${ }^{+}$Valores expresados en g $100 \mathrm{~g}^{-1}$ de $\mathrm{ms}$, DSH: diferencia significativa honesta, *: significativo $(\mathrm{P} \leq 0.05)$. 
Cuadro 5. Estructura genética de los cinco cruzamientos de mayor y los dos de menor contenido de aceite, proteína, almidón, cenizas y ácido fítico en grano.

\begin{tabular}{|c|c|c|c|c|c|c|c|}
\hline & \multirow{2}{*}{ Cruza } & \multirow{2}{*}{$\frac{Y_{i j}}{\left(\mathrm{~g} 100 \mathrm{~g}^{-1} \text { de } \mathrm{ms}\right)}$} & \multirow{2}{*}{$\mu$} & \multicolumn{2}{|c|}{ Núcleo } & \multicolumn{2}{|c|}{ Citoplasma } \\
\hline & & & & $g_{i}+g_{i}$ & $S_{i j}$ & $m_{i}-m_{i}$ & $r_{i i}$ \\
\hline & $\mathrm{L} 5 \times \mathrm{L} 1$ & 6.83 & & -0.263 & 0.842 & 0.073 & 0.87 \\
\hline & $\mathrm{L} 1 \times \mathrm{L} 3$ & 6.48 & & -0.061 & 0.321 & 0.169 & 0.744 \\
\hline & $\llcorner 4 \times\llcorner 5$ & 6.19 & & -0.109 & 0.295 & 0.405 & 0.298 \\
\hline \multirow[t]{7}{*}{ Aceite } & $\mathrm{L} 2 \times \mathrm{L} 5$ & 5.99 & 5.31 & -0.020 & 0.577 & -0.120 & 0.242 \\
\hline & $\mathrm{L} 4 \times \mathrm{L} 1$ & 5.93 & & 0.008 & 0.178 & 0.478 & 0.298 \\
\hline & $\mathrm{L} 3 \times \mathrm{L} 1$ & 4.65 & & -0.061 & 0.321 & -0.169 & -0.744 \\
\hline & $\mathrm{L} 2 \times \mathrm{L} 4$ & 4.61 & & 0.251 & -0.573 & -0.525 & 0.148 \\
\hline & $L 3 \times L 4$ & 8.95 & & 0.669 & -0.106 & 1.192 & -0.163 \\
\hline & $\mathrm{L} 2 \times \mathrm{L} 4$ & 8.87 & & 0.338 & -0.428 & 1.096 & 0.510 \\
\hline & $\mathrm{L} 3 \times \mathrm{L} 5$ & 8.53 & & 0.336 & 0.179 & 0.833 & -0.174 \\
\hline \multirow[t]{7}{*}{ Proteína } & $\llcorner 4 \times\llcorner 5$ & 8.29 & 7.36 & 0.321 & 0.543 & -0.359 & 0.432 \\
\hline & $\llcorner 5 \times\llcorner 4$ & 8.15 & & 0.321 & 0.543 & 0.359 & -0.432 \\
\hline & $\llcorner 4 \times L 1$ & 5.45 & & -0.347 & -0.383 & -1.093 & -0.085 \\
\hline & $\mathrm{L} 5 \times \mathrm{L} 1$ & 3.85 & & -0.68 & -1.667 & -0.734 & -0.423 \\
\hline & $\mathrm{L} 5 \times \mathrm{L} 2$ & 90.87 & & 0.308 & 4.559 & 0.473 & 2.543 \\
\hline & $\mathrm{L} 5 \times \mathrm{L} 4$ & 88.78 & & 1.352 & 4.398 & -1.142 & 1.190 \\
\hline & $\mathrm{L} 1 \times \mathrm{L} 5$ & 88.75 & & 3.086 & -0.200 & -1.455 & 4.334 \\
\hline \multirow[t]{7}{*}{ Almidón } & $\llcorner 4 \times\llcorner 5$ & 88.68 & 82.98 & 1.352 & 4.398 & 1.142 & -1.190 \\
\hline & $\llcorner 4 \times\llcorner 2$ & 87.76 & & -1.812 & 1.718 & 1.615 & 3.254 \\
\hline & $\mathrm{L} 2 \times \mathrm{L} 4$ & 78.02 & & -1.812 & 1.718 & -1.615 & -3.254 \\
\hline & $\mathrm{L} 3 \times \mathrm{L} 2$ & 73.37 & & -2.701 & -2.705 & -1.630 & -2.543 \\
\hline & $\mathrm{L} 5 \times \mathrm{L} 1$ & 1.85 & & -0.207 & 0.317 & -0.124 & 0.370 \\
\hline & $\mathrm{L} 1 \times \mathrm{L} 3$ & 1.83 & & 0.022 & -0.008 & 0.106 & 0.211 \\
\hline & $\mathrm{L} 2 \times \mathrm{L} 4$ & 1.82 & & 0.139 & -0.123 & 0.101 & 0.208 \\
\hline \multirow[t]{7}{*}{ Cenizas } & $\mathrm{L} 1 \times \mathrm{L} 2$ & 1.79 & 1.50 & 0.017 & 0.109 & -0.008 & 0.178 \\
\hline & $\mathrm{L} 2 \times \mathrm{L} 5$ & 1.71 & & -0.100 & 0.163 & 0.132 & 0.024 \\
\hline & $\mathrm{L} 5 \times \mathrm{L} 4$ & 0.90 & & -0.085 & -0.278 & -0.031 & -0.201 \\
\hline & $\mathrm{L} 5 \times \mathrm{L} 3$ & 0.70 & & -0.095 & -0.540 & -0.018 & -0.145 \\
\hline & $\mathrm{L} 1 \times \mathrm{L} 2$ & 2.75 & & 0.048 & 0.192 & 0.183 & 0.262 \\
\hline & $\llcorner 4 \times\llcorner 1$ & 2.36 & & 0.163 & 0.100 & 0.058 & -0.032 \\
\hline & $\mathrm{L} 5 \times \mathrm{L} 1$ & 2.33 & & -0.055 & 0.024 & -0.144 & 0.440 \\
\hline \multirow[t]{4}{*}{ Ácido Fítico } & $\mathrm{L} 1 \times \mathrm{L} 4$ & 2.30 & 2.07 & 0.163 & 0.100 & -0.058 & 0.032 \\
\hline & $\mathrm{L} 1 \times \mathrm{L} 3$ & 2.22 & & -0.046 & 0.067 & -0.015 & 0.146 \\
\hline & $\llcorner 5 \times\llcorner 4$ & 1.45 & & 0.034 & -0.348 & -0.202 & -0.097 \\
\hline & $\mathrm{L} 5 \times \mathrm{L} 3$ & 1.21 & & -0.175 & -0.249 & -0.159 & -0.275 \\
\hline
\end{tabular}

$Y_{i j}$ : valor fenotípico observado de la cruza $(i, j), \mu$ : media general, $g_{i}$ y $g_{j:}$ efecto de la ACG del progenitor $i$ y $j$, respectivamente, $s_{i j}$ : efecto de ACE de la cruza $(i, j), m_{i}-m_{i}$ : efecto materno del progenitor $i$ y $j$, respectivamente, $r_{i j:}$ efecto recíproco de cruzas. 
por hibridación y otros por selección para la formación de variedades.

\section{CONCLUSIONES}

Se encontraron efectos significativos de aptitud combinatoria general (ACG) y específica (ACE) en la mayoría de las características bioquímicas estudiadas; sin embargo, los efectos de ACE fueron los de mayor contribución a la variación genética expresada. Se demostró que existe potencial genético para influir en la producción de maíz de alta calidad alimenticia a mediano y largo plazo al aprovechar las líneas y las cruzas específicas, las cuales pueden utilizarse ya sea en la formación de híbridos, variedades sintéticas, o en el establecimiento de un programa de mejoramiento por selección recurrente. Se presentaron efectos maternos para la mayoría de las variables estudiadas, por lo que para el mejor aprovechamiento de las líneas es necesario considerar la dirección de las cruzas formadas entre ellas.

\section{BIBLIOGRAFÍA}

AACC, American Association of Cereal Chemists (1999a) Aproved Methods of Analysis. 11th edition. Method 30-25.01 Crude Fat in Wheat, Corn, and Soy Flour, Feeds, and Mixed Feeds. AACC International. St. Paul, Minnesota, USA. https://doi.org/10.1094/ AACCIntMethod-30-25.01

AACC, American Association of Cereal Chemists (1999b) Aproved Methods of Analysis. 11th edition. Method 46-11.02 Crude Protein Improved Kjeldahl Method, Copper Catalyst Modification. AACC International. St. Paul, Minnesota, USA. https://doi. org/10.1094/AACCIntMethod-46-11.02

AACC, American Association of Cereal Chemists (1999c) Aproved Methods of Analysis. 11th edition. Method 76-13.01, Total Starch Assay Procedure (Megazyme Amyloglucosidase/alpha-Amylase Method). AACC International. St. Paul, Minnesota, USA. https:// doi.org/10.1094/AACCIntMethod-76-13.01

AACC, American Association of Cereal Chemists (1999d) Aproved Methods of Analysis. 11th edition. Method 08-01.01 Ash - Basic Method AACC International. St. Paul, Minnesota, USA. https://doi. org/10.1094/AACCIntMethod-08-01.01

Ávila P. M. A., S. A. Rodríguez H., M. E. Vázquez B., F. Borrego E., A. J. Lozano R. y A. López B. (2009) Aptitud combinatoria y efectos recíprocos en líneas endogámicas de maíz de Valles Altos del centro de México. Agricultura Técnica en México 35:285-293.

Bisen P., A. Dadheech, N. Namrata, A. Kumar, G. Solanki and T. R Dhakar (2017) Combining ability analysis for yield and quality traits in single cross hybrids of quality protein maize (Zea mays L.) using diallel mating design. Journal of Applied and Natural Science 9:1760-1766, https://doi.org/10.31018/jans.v9i3.1435

Brunt K., P. Sanders and T. Rozema (1998) The enzymatic determination of starch in food, feed and raw materials of the starch industry. Starch 50:413-419, https://doi.org/10.1002/(SICI)1521379X(199810)50:10<413::AID-STAR413>3.0.CO;2-F

Cázares-Sánchez E., J. L. Chávez-Servia, Y. Salinas-Moreno, F. CastilloGonzález y P. Ramírez-Vallejo (2015) Variación en la composición del grano entre poblaciones de maíz (Zea mays L.) nativas de Yucatán, México. Agrociencia 49:15-30.

Cervantes-Ortiz F., G. García-De los Santos, A. Carballo-Carballo, D. Bergvinson, J. L. Crossa, M. Mendoza-Elos y E. Moreno-Martínez (2007) Herencia del vigor de plántula y su relación con caracteres de planta adulta en líneas endogámicas de maíz tropical. Agrociencia $41: 425-433$.
Dragičević V. D., S. D. Sredojević, V. A. Perić, A. R. Nišavić and M. B. Srebrić (2011) Validation study of a rapid colorimetric method for the determination of phytic acid and inorganic phosphorus from seeds. Acta Periodica Tecnologica 42:11-21, https://doi. org/10.2298/APT1142011D

Escorcia-Gutiérrez N., J. D. Molina-Galán, F. Castillo-González y J. A. Mejía-Contreras (2010) Rendimiento, heterosis y depresión endogámica de cruzas simples de maíz. Revista Fitotecnia Mexicana 33:271-279.

Esquivel E. G., F. Castillo G., J. M. Hernández C., A. Santacruz V., G. García S. y J. A. Acosta G. (2013) Aptitud combinatoria en maíz con divergencia genética en el Altiplano mexicano. Revista Mexicana de Ciencias Agrícolas 4:5-18.

Figueroa C. J. D., D. E. Narváez G., A. Mauricio S., S. Taba, M. Gaytán M., J. J. Vélez M, F. Rincón S. y F. Aragón C. (2013) Propiedades físicas del grano y calidad de los grupos raciales de maíces nativos (criollos) de México. Revista Fitotecnia Mexicana 36:305-314.

Galicia L., A. Miranda, M. G. Gutiérrez, O. Custodio, A. Rosales, N. Ruiz, R Surles y N. Palacios (2012) Protocolos de Laboratorio. Laboratorio de Calidad Nutricional de Maíz y Análisis de Tejido Vegetal CIMMYT. México, D. F. 50 p.

Griffing B. (1956) Concept of general and specific combining ability in relation to diallel crossing systems. Australian Journal of Biological Sciences 9:463-493, https://doi.org/10.1071/ BI9560463

Guerrero-Guerrero C., A. Espinoza-Banda, A. Palomo-Gil, E. Gutiérrez-Del Río J. G. Luna-Ortega y N. Rodríguez-Dimas (2012) Comportamiento genético y aptitud combinatoria en cruzas simples con líneas élite de maíz. Universidad y Ciencia 28:65-77.

Hallauer A. R., M. J. Carena and J. B. Miranda F. (2010) Quantitative Genetics in Maize Breeding. Springer. New York, USA. 663 p.

Kirk R. S., R. Sawyer y H. Egan (1996) Composición y Análisis de Alimentos de Pearson. $2^{a}$ ed., Compañía Editorial Continental. México, D. F. $777 \mathrm{p}$.

Long J. K., M. Bänzinger and M. E. Smith (2004) Diallel analysis of grain iron and zinc density in Southern African-adapted maize inbreds. Crop Science 44:2019-2026, https://doi.org/10.2135/ cropsci2004.2019

Mahgoub M. G. (2011) Partitioning of general and specific combining ability effects for estimating maternal and reciprocal effects Journal of Agricultural Science 3:213-222, https;//doi org/10.5539/jas.v3n2p213

Martínez G. A. (1983) Diseños y Análisis de Experimentos de Cruzas Dialélicas. Centro de Estadística y Cálculo. Colegio de Postgraduados. Chapingo, Estado de México. 252 p.

Picón-Rico R., R. E. Preciado-Ortiz, F. Cervantes-Ortiz, J. Covarrubias-Prieto y A. Terrón-Ibarra (2018) Efectos heteróticos en líneas doble haploides de maíz de grano blanco y alto contenido de aceite. Revista Fitotecnia Mexicana 41:177-186.

Qamar S., M. Aslam and M. A. Javed (2016) Determination of proximate chemical composition and detection of inorganic nutrients in maize (Zea mays L.). Materials Today: Proceedings 3:715-718, https://doi.org/10.1016/j.matpr.2016.01.118

Raboy V., K. A. Young, J. A. Dorsch and A. Cook (2001) Genetics and breeding of seed phosphorus and phytic acid. Journal of Plant Physiology 158:489-497, https://doi.org/10.1078/0176-161700361

Sánchez-Ramírez F. J., M. C. Mendoza-Castillo, M. Mendoza-Rodríguez, F. Castillo-González, S. Cruz-Izquierdo, S. Castro-Nava y J. D. MolinaGalán (2017) Aptitud combinatoria de líneas endogámicas para la producción de híbridos de maíz (Zea mays L.) de cruza simple en condiciones de riego. Agrociencia 51:393-407.

SAS Institute (2003) SAS® 9.1 User's Guide: Statistics. Statistical Analysis System Institute. Cary, North Carolina, USA. 694 p.

Serna-Saldívar S. O., J. A. Gutiérrez-Uribe, S. Mora-Rochin y S. García-Lara (2013) Potencial nutraceútico de los maíces criollos y cambios durante el procesamiento tradicional y con extrusión. Revista Fitotecnia Mexicana 36:295-304

SIAP, Servicio de Información Agroalimentaria y Pesquera (2017) Anuario estadístico de la producción agrícola. Servicio de Información Agroalimentaria y Pesquera. SADER. Ciudad de México. https:// nube.siap.gob.mx/cierreagricola/ (Marzo, 2019). 
Sprague G. F. and L. A. Tatum (1942) General vs. specific combining ability in single crosses of corn. Journal of the American Society of Agronomy 34:923-932.

Vats P. and U. C. Banerjee (2004) Production studies and catalytic properties of phytases (myo-inositolhexakisphosphate phosphohydrolases): an overview. Enzyme and
Microbial Technology 35:3-14, https://doi.org/10.1016/j. enzmictec. 2004.03.010

Vázquez C. M. G., H. Mejía A., C. Tut C., N. Gómez M. (2012) Características de granos y tortillas de maíces de alta calidad proteínica desarrollados para los valles altos centrales de México. Revista Fitotecnia Mexicana 35:23-31. 